\title{
Study of the radio and optical properties of Active Galactic Nuclei
}

\author{
H. V. Abrahamyan * \\ NAS RA Byurakan Astrophysical Observatory (BAO), Byurakan 0213, Aragatzotn Province, Armenia
}

\begin{abstract}
The investigation of the physical properties of active galactic nuclei is one of the most important tasks of astronomy. Until now, the properties of AGN have not been fully studied. Therefore, we have studied a number of important properties of AGN. For example, one of the main properties of AGN is the variability. We have studied samples of AGN derived by different methods. For these sources, we have identified some properties of activity for the radio and optical ranges. These properties give us some insight into the active galaxies.
\end{abstract}

Keywords: active galactic nuclei, QSO, radio spectral index, cross-correlation

\section{Introduction}

During the first half of the 20th century, photographic observations of nearby galaxies detected some characteristic signatures of AGN emission, although there was not yet a physical understanding of the nature of the AGN phenomenon. Some early observations included the first spectroscopic detection of emission lines from the nuclei of NGC 1068 and Messier 81 by Edward Fath (published in 1909), and the discovery of the jet in Messier 87 by Heber Curtis (published in 1918). Further spectroscopic studies by astronomers including Vesto Slipher, Milton Humason, and Nicholas Mayall noted the presence of unusual emission lines in some galaxy nuclei. In 1943, Carl Seyfert published a paper in which he described observations of nearby galaxies having bright nuclei that were sources of unusually broad emission lines. Galaxies observed as part of this study included NGC 1068, NGC 4151, NGC 3516, and NGC 7469. Active galaxies such as these are known as Seyfert galaxies in honor of Seyfert's pioneering work.

The development of radio astronomy was a major catalyst to understanding AGN. Some of the earliest detected radio sources are nearby active elliptical galaxies such as Messier 87 and Centaurus A. Another radio source, Cygnus A, was identified by Walter Baade and Rudolph Minkowski as a tidally distorted galaxy with an unusual emission-line spectrum, having a recessional velocity of 16,700 kilometers per second. The 3C radio survey led to further progress in discovery of new radio sources as well as identifying the visible-light sources associated with the radio emission. In photographic images, some of these objects were nearly point-like or quasi-stellar in appearance, and were classified as quasi-stellar radio sources (later abbreviated as "quasars").

Soviet Armenian astrophysicist Viktor Ambartsumian introduced Active Galactic Nuclei in the early 1950s. At the Solvay Conference on Physics in 1958, Ambartsumian presented a report arguing that "explosions in galactic nuclei cause large amounts of mass to be expelled. For these explosions to occur, galactic nuclei must contain bodies of huge mass and unknown nature. From this point forward Active Galactic Nuclei (AGN) became a key component in theories of galactic evolution." His idea was initially accepted skeptically.

A major breakthrough was the measurement of the redshift of the quasar 3C 273 by Maarten Schmidt, published in 1963. Schmidt noted that if this object was extragalactic (outside the Milky

*abrahamyanhayk@gmail.com 
Way, at a cosmological distance) then its large redshift of 0.158 implied that it was the nuclear region of a galaxy about 100 times more powerful than other radio galaxies that had been identified. Shortly afterward, optical spectra were used to measure the redshifts of a growing number of quasars including 3C 48 , even more distant at redshift 0.37 .

The enormous luminosity of these quasars as well as their unusual spectral properties indicated that their power source could not be ordinary stars. Accretion of gas onto a supermassive black hole was suggested as the source of quasars' power in papers by Edwin Salpeter and Yakov Zeldovich in 1964. In 1969 Donald Lynden-Bell proposed that nearby galaxies contain supermassive black holes at their centers as relics of "dead" quasars, and that black hole accretion was the power source for the non-stellar emission in nearby Seyfert galaxies. In the 1960s and 1970s, early X-ray astronomy observations demonstrated that Seyfert galaxies and quasars are powerful sources of X-ray emission, which originates from the inner regions of black hole accretion disks.

Today, AGN are a major topic of astrophysical research, both observational and theoretical. AGN research encompasses observational surveys to find AGN over broad ranges of luminosity and redshift, examination of the cosmic evolution and growth of black holes, studies of the physics of black hole accretion and the emission of electromagnetic radiation from AGN, examination of the properties of jets and outflows of matter from AGN, and the impact of black hole accretion and quasar activity on galaxy evolution.

Many active galaxies, especially active galactic nuclei (AGN), are strong in radio wavelengths (e.g. many objects in Véron-Cetty \& Véron (2010) have strong radio; the catalogue of Blazars by Massaro et al., 2015 is complied exclusively from objects having radio detection); hence studying radio emission from galaxies may be a key to identify the active ones among them. Radio galaxies, quasars, blazars, megamasers and other classes of objects are strong radio emitters. Radio galaxies and their relatives, radioloud quasars and blazars, are types of AGN that are very luminous at radio wavelengths, with luminosity up to $10^{39} \mathrm{~W}$ in the range of $10 \mathrm{MHz}$ to $100 \mathrm{GHz}$. This radio emission is due to the synchrotron process; the observed radio structure is determined by the interaction between two opposite jets and the external medium, affected by relativistic beaming. The host galaxies are almost exclusively giant elliptical galaxies and radio galaxies can be detected at large distances, making them valuable tools for observational cosmology. Recently, much work was done related to the effects of these objects on the intergalactic medium as well, particularly in galaxy groups and clusters.

\section{Creation of Cross-Correlation program: The IRAS PSC/FSC Com- bined Catalogue}

To study the IR point sources we took IRAS PSC and FSC catalogues. They were created in 1986 (IRAS PSC) and 1989 (IRAS FSC), and provide information of fluxes at wavelengths 12, 25, 60 and $100 \mu \mathrm{m}$. IRAS PSC contains 245,889 sources and IRAS FSC contains 173,044 sources at galactic latitude $|\mathrm{b}|>10^{\circ}$. Each source in these catalogues has coordinate errors. We did a cross-correlation between IRAS PSC and FSC and considered errors for each source; we took those identifications having positional errors between the sources not exceeding $3 \sigma$ (which corresponds to $99.73 \%$ probability). For that, in frame of the Armenian Virtual Observatory (ArVO), we created a software through which we made cross-correlations. To obtain information about fluxes in other IR bands we did cross-correlation using the same method with AKARI-IRC All-Sky Survey Point Source Catalogue, AKARI-FIS All-Sky Survey Bright Source Catalogue and used VizieR for WISE catalogue having very accurate positions.

In catalogues IRAS-PSC and IRAS-FSC, for each source we have positional errors given as Minor and Major axes, which relate to the orientation of the satellite during the observations. For crosscorrelations, we used Major and average axes positional errors for each object. We created a software through which we made cross-correlations. This software allows considering positional errors for each source individually and we have taken identifications having coordinate differences between counterparts not exceeding $3 \sigma$ (calculated using both sigmas from PSC and FSC). As a result, we obtained 73,770 identifications when using the Major axes and 72,777 when using the average errors. 
To avoid losing identifications, finally we build our Catalogue using identification with Major axes (Abrahamyan et al. (2015)). Some sources have two or more associations. For these sources we take associations using the following criteria:

- the first (nearest by distance) association is taken, if the second one (and others) is 3 times farther than the first one (these are the best identifications and we call them Category 1). We have $58,296(79 \%)$ such associations.

- in case of positional ambiguity (when the genuine association is not clear as in Category 1), we take those associations having close fluxes (coincidence within 20\%) and quality flags indicating the same nature of objects. We call them Category 2 associations and we have 10,488 (14\%) such cases.

- the first (nearest by distance) association is taken, if the second one (and others) is 2 times farther than the first one (weaker criterion giving worst identifications). We call them Category 3 associations and we have 4901 (7\%) such cases.

We are left with 85 worse associations, which also may be regarded as genuine ones with weaker criteria. We have built a distribution of the number of objects from distances of their identifications. Figure 1 shows that the majority of identified objects have limited distance and we have derived by interpolation with polynomial fit that 73.4 arcsec should be taken as the radius of reliable associations.

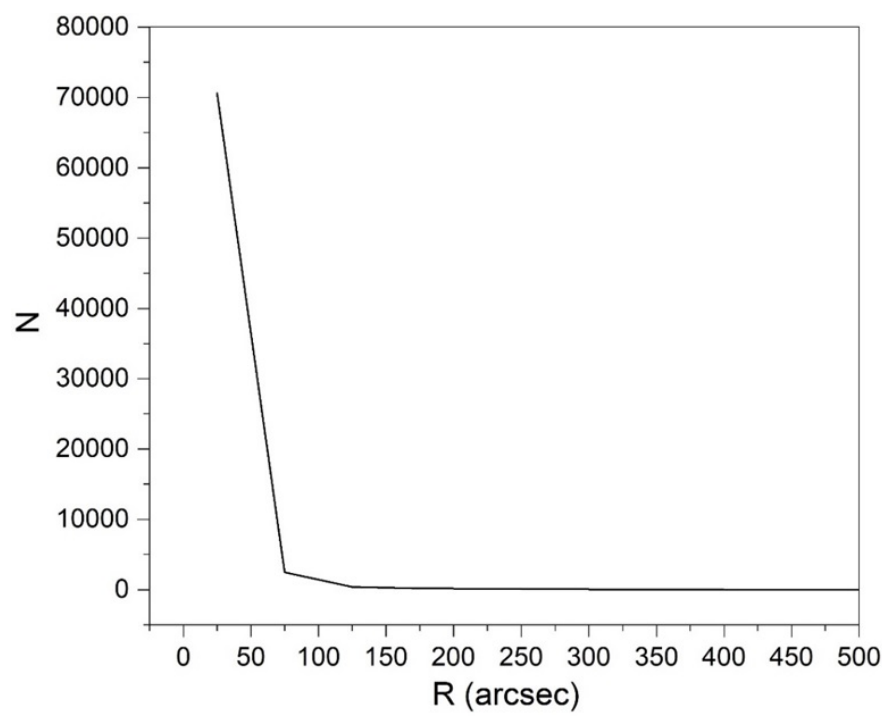

Figure 1. Distribution of the number of sources by distances of their identifications (IRAS-PSC and IRAS-FSC)

For cross-correlations with IRAS catalogues most astronomers use search radius $60^{\prime \prime}$. However, as we see from the comparison and analysis of these identification tools, if we take $60^{\prime \prime}$ for crosscorrelations, then we lose many genuine associations.

For our IRAS PSC/FSC Combined Catalogue we give the probability of classification for each source into "star" or "galaxy". For this purpose we used fluxes and quality flags from IRAS and other catalogues. If IRAS source is confidently identified with AKARI-IRC and there is no match in AKARIFIS, then in all probabilities the object is a star, and if an IRAS source is detected in AKARI-FIS without a record in AKARI-IRC, then in all probabilities the object is a galaxy. For brighter sources, when all records are available, we use the IR colours, i.e. we follow the change of the flux from shorter to longer wavelengths; in case of a decrease it is a high probability star and in case of an increase it is a high probability galaxy. We can in fact estimate the type of all sources based on IRAS flux and quality flag data, as well as on AKARI and WISE/2MASS measurements. If all data show the same type of object, then we give it as a genuine one, and if there is a ambiguity, we give the most probable 
type with a flag. In Figure 2, for a possible star and a galaxy we built spectral energy distributions (SED) based on our collected data from NIR 2MASS JHK to FIR IRAS and AKARI. The star is IRAS $00012+7614=$ IRAS F00012+7614 and the galaxy is IRAS F00041-3446. These sources were chosen as examples because they are very typical for stars and galaxies. The star is TYC 4492-1689-1 $(\mathrm{V}=10.43)$ and the source chosen as a galaxy is also the radiosource NVSS J000639-342943.
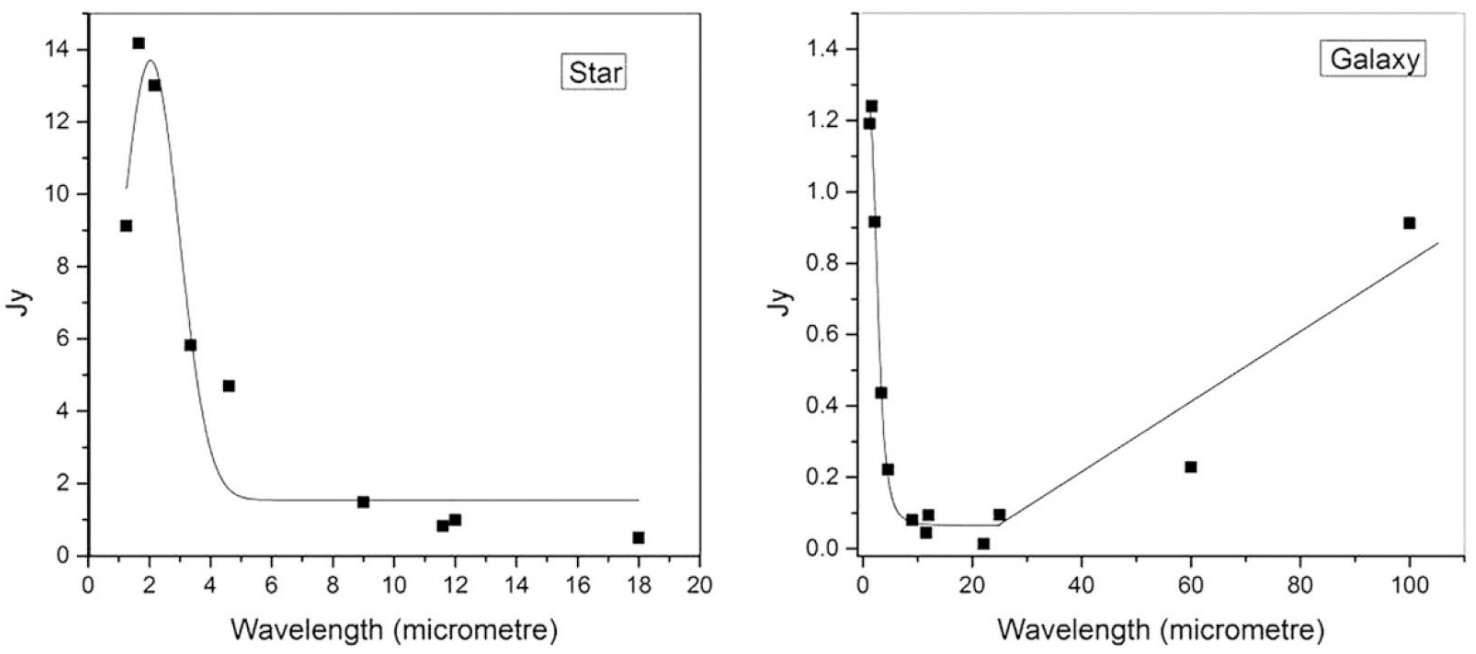

Figure 2. "Star" (IRAS 00012+7614=IRAS F00012+7614) and "Galaxy" (IRAS F00041-3446) SEDs based on 2MASS, WISE, AKARI and IRAS photometric data.

We have created a software through which we made cross-correlations with search radius for each source individually based on its positional error, taking only those associations having positional distance between sources not exceeding $3 \sigma$. We have created the IRAS PSC/FSC Combined Catalogue to show the efficiency of the software and to have a joint IRAS catalogue for further statistical studies and investigations of individual sources. IRAS PSC/FSC contains 345,163 sources, including 73,770 common ones from both catalogues (IRAS-PSC and IRAS-FSC). We calculated the improved coordinates for these 73,770 sources, as well as individual positional errors based on shifts of IRAS-PSC and IRAS-FSC positions. We also have calculated improved Minor and Major axes coordinates errors.

\section{Radio variable sources at $1400 \mathrm{MHz}$ and their optical variability}

NVSS and FIRST radio catalogues have been cross-matched. Our principle is to take into account positional errors for individual sources, and we have applied similar to our previous research method (Abrahamyan et al. (2015)). In the FIRST catalogue there is no information on positional errors for each source, that is why 5 arcsecond as errors for all sources is adopted. In NVSS catalogue, each source is given with its individual positional error. We have created a software through which crosscorrelations are done. This software allows considering positional errors for each source individually and we have taken associations having coordinate differences between counterparts not exceeding $3 \sigma$ (calculated using both $\sigma$-s from NVSS and FIRST). As a result, we have obtained 556,282 associations between NVSS and FIRST (Abrahamyan et al. (2018)).

Our main task is the revelation of the variability of radio sources in radio wavelengths. For variability criteria, we will take into account those radio sources which have associations within less than $3 \sigma$ of the positional errors and for which the second association is 2 times farther than the first one. The systematic shift (SS) between fluxes of NVSS and FIRST catalogues was considered. We counted SS between these catalogues to get rid of systematic errors that could appear due to different flux calibration. As FIRST accuracy is higher, we have shifted NVSS using SS. The first step that was accomplished is computing systematic shift (SS) for fluxes between NVSS and FIRST (SS $=-0.765$ mJy). 


$$
\Delta F=\left|F_{F I R S T}-\left(F_{N V S S}-S S\right)\right|-3 \sigma
$$

where $\sigma$ is the combined flux error:

$$
\sigma=\sqrt{\left(\text { Error }_{F I R S T}^{2}+\text { Error }_{N V S S}^{2}\right)}
$$

We have carried out a cross-correlation of NVSS and FIRST catalogues to distinguish sources which have large differences of fluxes at $1400 \mathrm{MHz}$. We have selected 6301 radio sources with flux difference at least $15 \mathrm{mJy}$. Further investigation of these radio sources led to a new sample of radio sources, which have high optical variability.

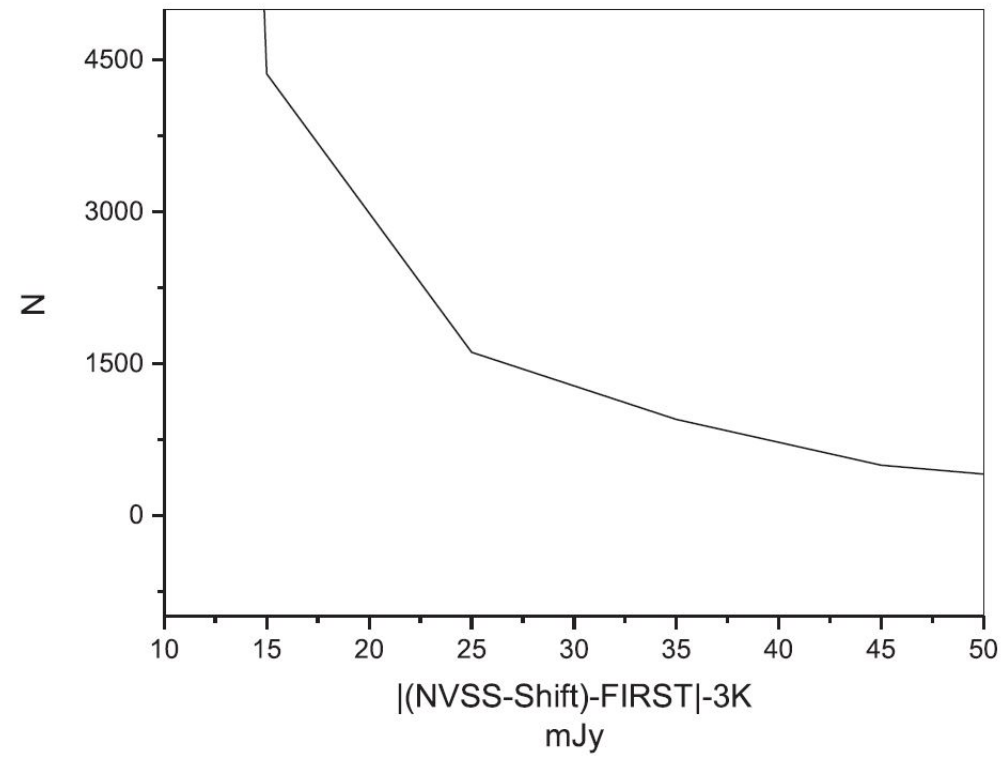

Figure 3. $\Delta \mathrm{F}$ distribution showing breaks at 15,25 and $45 \mathrm{mJy}$.

\begin{tabular}{|c|c|c|}
\hline $\mathrm{N}_{o}$. & Activity type & Numbers \\
\hline 1 & Blazar (BZB, BZG, BZQ, BZU) & 176 \\
\hline 2 & QSO & 333 \\
\hline 3 & Sy $1.0 /$ Sy 1 & 9 \\
\hline 4 & Sy 1.5 & 6 \\
\hline 5 & Sy 2.0 / Sy 2 & 5 \\
\hline 6 & AGN & 45 \\
\hline 7 & FSS (Flat-Spectrum radio source) & 41 \\
\hline \multirow[t]{4}{*}{8} & USS (Ultra-Steep-Spectrum radio source) & 4 \\
\hline & Known (total) & $619(25.5 \%)$ \\
\hline & Unknown & $1806(74.5 \%)$ \\
\hline & Total & $2425(100 \%)$ \\
\hline
\end{tabular}

\begin{tabular}{cll}
\multicolumn{3}{l}{ Activity types of 6301 radio sources having radio variability. } \\
\hline $\mathrm{N}_{0 .}$ & Activity type & Numbers \\
\hline 1 & Blazar (BZB, BZG, BZQ BZU) & 308 \\
2 & QSO & 639 \\
3 & Sy $1.0 /$ Sy 1 & 19 \\
4 & Sy 1.2 & 2 \\
5 & Sy 1.5 & 6 \\
6 & Sy 1.9 & 2 \\
7 & Sy $2.0 /$ Sy 2 & 9 \\
8 & AGN & 97 \\
9 & Starburst & 1 \\
10 & FSS (Flat-Spectrum radio source) & 87 \\
11 & USS (Ultra-Steep-Spectrum radio source) & 36 \\
\hline & Known (total) & $\mathbf{1 2 0 6}(\mathbf{1 9 \%})$ \\
& Unknown & $\mathbf{5 0 9 5}(\mathbf{8 1 \%})$ \\
& Total & $\mathbf{6 3 0 1}(\mathbf{1 0 0 \%})$ \\
\hline
\end{tabular}

Figure 4. 6301 radio sources

\section{Optical variability of blazars}

To understand some properties of blazars, we used Roma Multifrequency Catalog of Blazars (BZCAT) 5th version. Altogether, 3,561 objects are given as BZB, BZQ, BZG, or BZU corresponding to BLL, FSR quasars, galaxies, and blazars of uncertain/transitional type (Abrahamyan et al. (2019)). Having 3,561 blazars that have radio variability, we try to check how many of these sources are optically variable. We cross-correlated these radio sources with POSS1-based and POSS2-based optical 
catalogs: APM, USNO A2.0, USNO B1.0, and GSC 2.3.2. In Figure 5, we give graphs of the absolute magnitude versus redshift. Basically, three separated areas (BZB, BZG, and BZQ) are observed. Three types of blazars (BZB, BZG, and BZQ) are mostly separated, but there is overlap with each other to some extent.
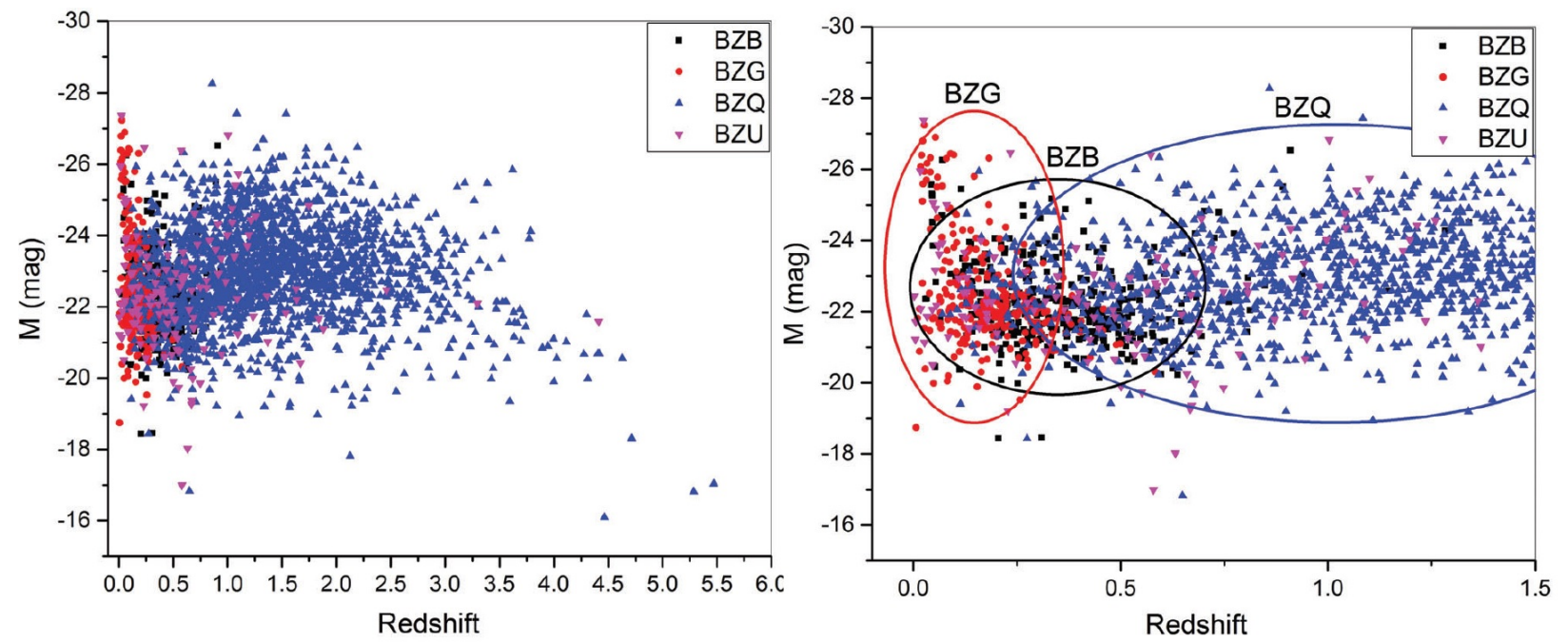

Figure 5. Absolute magnitude vs. redshift

Thus, we have 2,121 radio sources that have optical variability. For each source,we have four means for understanding their variability and for each source, based on this, we give variability category flags from 0 to 3 For a detailed picture of variability of these sources, we counted 4 category flags together.

\begin{tabular}{|llll|}
$\begin{array}{l}\text { Summarizing number } \\
\text { for four category flags } \\
\text { (all possible configurations } \\
\text { of the flags) }\end{array}$ & $\begin{array}{l}\text { Number of variable } \\
\text { sources }\end{array}$ & Total & Comments \\
\hline 3333 & 23 & 51 & Extreme variability \\
\hline 3332 & 10 & & \\
\hline 3322,3331 & 18 & 126 & High variability \\
\hline $3330,3321,3222$ & 23 & & \\
\hline $3221,3311,2222$ & 42 & 483 & Medium variability \\
\hline $3310,3220,3211$ & 61 & & \\
\hline $3300,3210,3111,2220$ & 122 & & \\
\hline $3200,3110,2210,2111$ & 118 & 1,461 & Low variability \\
\hline $2200,2110,1111$ & 243 & & \\
\hline $3000,2100,1110$ & 299 & & \\
\hline 2000,1100 & 670 & & \\
\hline 1000 & 492 & & \\
\hline Total & & & \\
\hline
\end{tabular}

Figure 6. Number of optically variable radio sources based on their variability categories and flags (0-have not variability, 1-low variability, 2-medium variability, and 3-high variability)

We have obtained 2,121 blazars that have optical variability using POSS1 and POSS2 epoch measurements. We have compared our work with the one reported by Hovatta et al. (2014). In the work, the authors investigated optical data from the PTF and the CRTS to study the variability of $\gamma$-ray-detected and non detected objects in a large population of AGN selected from the Candidate Gamma-Ray Blazar Survey and Fermi Gamma-Ray Space Telescope catalogs. Their samples include 714 sources with PTF data and 1,244 sources with CRTS data. We compared our list with the list by Hovatta et al. (2014). As a result, we obtained 704 identified sources. 


\section{The nature of active galaxies based on their radio properties}

We use data from Véron-Cetty \& Véron (2010) catalog (VCV-13). This catalog includes 133,336 quasars, 1,374 BL Lac objects, and 34,231 active galaxies (including 16,517 Seyfert 1.0). We have considered 34,231 active galaxies for our research (Abrahamyan (2020) and Abrahamyan et al. (2020)).

For investigation, galaxies with magnitudes in the range of $12^{m}-19^{m}$ have been taken. In the next step, we have cross-correlated (Abrahamyan et al. (2015)) these objects with radio catalogs: FIRST, NVSS, 87GB, GB6, 3C, 4C, 7C, 8C, 9C, 10C, SUMSS, WISH, WENSS, Molonglo Reference Catalogue of Radio Sources, Texas Survey of radio sources at $365 \mathrm{MHz}$, Miyun $232 \mathrm{MHz}$ survey, CLASS survey of radio sources, $74 \mathrm{MHz}$ VLA Low-frequency Sky Survey Redux, and the GMRT $150 \mathrm{MHz}$ all-sky radio survey. As a result, we have 4,437 objects that have been radio-identified. 4,437 objects have 1-10 radio fluxes at different wavelengths. In this work, radio catalogs that cover the $38 \mathrm{MHz}$ to $15.7 \mathrm{GHz}$ frequency range have been used. For our investigation, we have taken objects that have six or more radio fluxes at different wavelengths. With six and more radio fluxes, there is an opportunity to better understand some physical properties in radio. So, we have 198 objects with six or more radio fluxes.

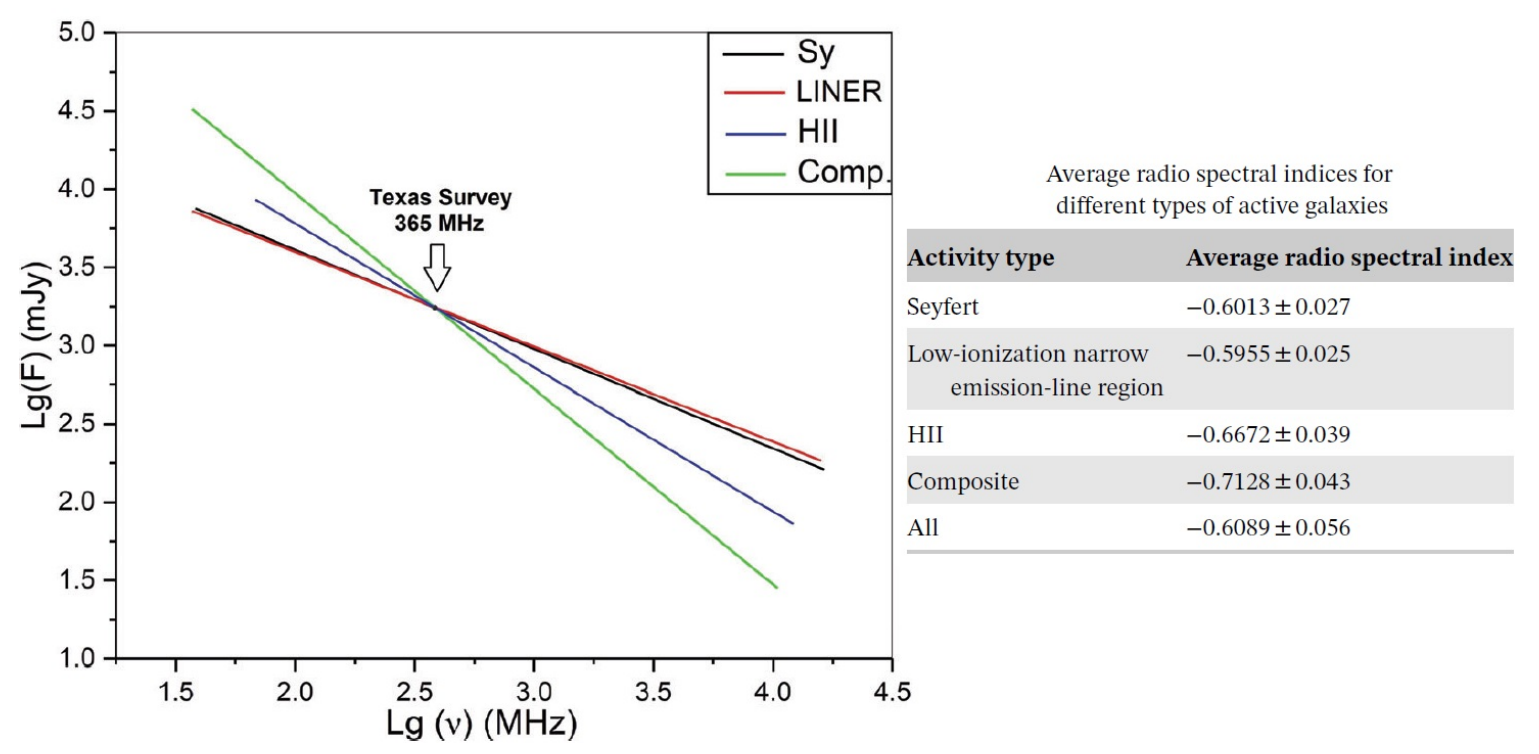

Figure 7. Average radio spectra for our object

Active galaxies are very interesting objects in the Universe. In order to understand some physical properties, we must identify which properties our objects have in radio range. We have 198 active galaxies with six or more radio fluxes at different wavelengths. A very important radio property for radio objects is the radio spectral index. It shows steep radio spectra. Using six or more frequencies, we have developed a graph for all 198 galaxies (lg[flux] vs. $\lg [$ frequencies]). Using an $\lg [$ flux] versus $\lg$ [frequencies] graph for each source, we have made linear fitting. The software "Origin" gives the formula for each linear fit, and using that, we have measured the radio spectral index for each source. The plot shows steep radio spectra for each line, and that is considered radio spectral index. As examples, we give average radio spectra for our objects in Figure 7.

Using redshift information, we have estimated physical sizes for our objects. We have eight objects that have HII activity. For these sources, we have also estimated physical sizes, and for one of them, we have obtained very big physical size $(832.2 \mathrm{kpc})$ compared to the other HII objects, which are up to $116.35 \mathrm{kpc}$. With the purpose of calculating the average size and drawing other comparisons, we have excluded this object. Therefore, using physical sizes, we have developed the dependence of radio spectral index on physical size (Figure 8). 


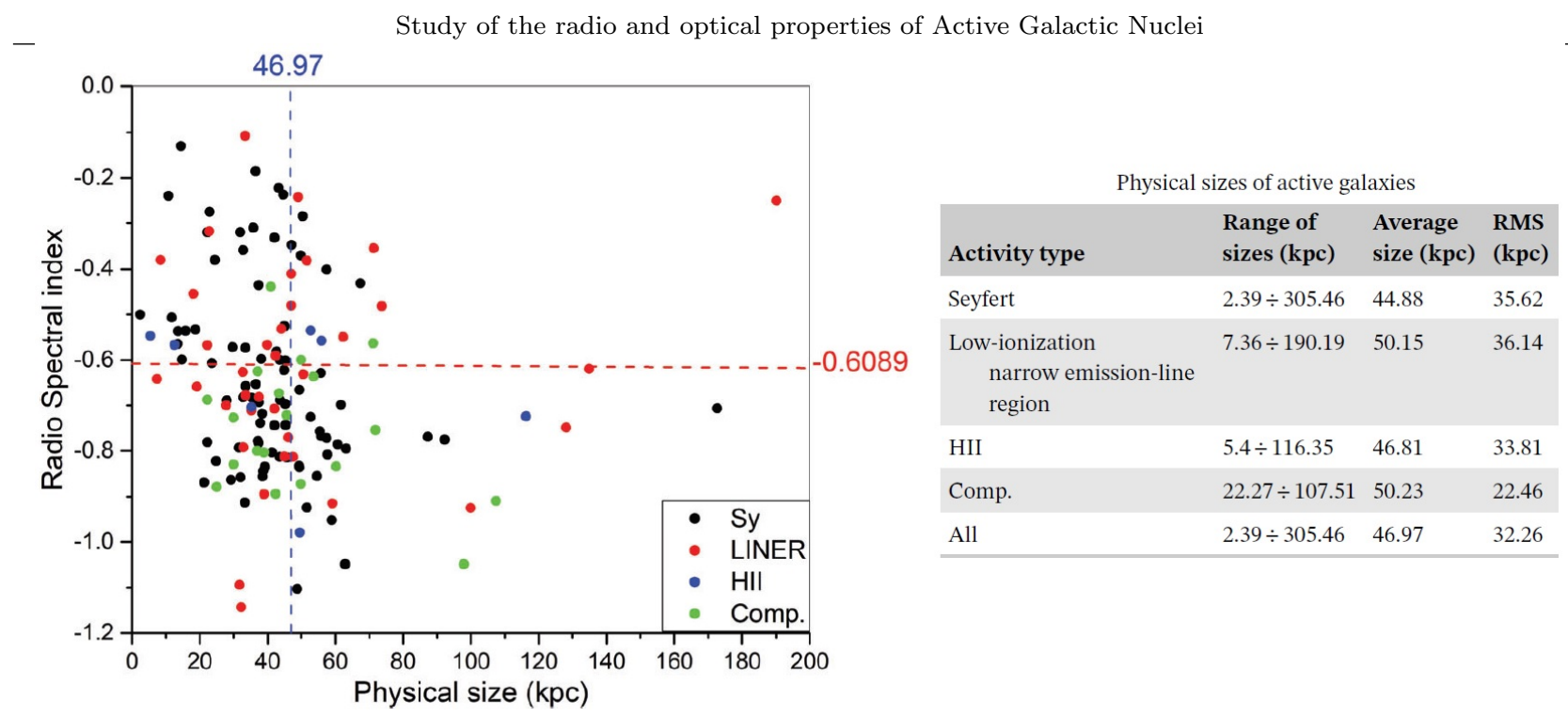

Figure 8. Radio spectral index versus physical size

\section{Result and conclusion}

Optical identifications of a few thousands of IRAS sources showed that IRAS Point Source and IRAS Faint Source catalogues contain many quasars and active galactic nuclei, late-type stars, planetary nebulae, variables, etc. To increase the efficiency of using IRAS PSC and FSC, which contain a lot of common sources, one needs a joint catalogue of all IRAS point sources with improved data based on both catalogues. However, cross-correlation of the catalogues is not so easy, as the association of many sources is relative, and not always it is obvious, which source from one catalogue corresponds to the other one in the second catalogue. This problem exists in case of using standard cross-correlation tools like VizieR. Therefore, we have created a tool for cross-matching astronomical catalogues and we have applied it to IRAS PSC and FSC. Using this tool we have carried out identifications with a search radius corresponding to $3 \sigma$ of errors for each source individually rather than a standard radius for all sources. As a result, we obtained 73,770 associations. We showed that in case of cross-correlation of these catalogues by VizieR, we had to take 161.95 arcseconds radius not to lose any association; however, in this case a lot of false associations appear for many sources. In addition, we have made cross-correlations with AKARI-IRC, AKARI-FIS and WISE catalogues. As a result we created a catalogue with high positional accuracy and with 17 photometric measurements from 1.25 to $160 \mu \mathrm{m}$ range, providing a detailed catalogue for IRAS point sources (Abrahamyan et al. (2015)).

In the next work we have cross-correlated NVSS and FIRST radio catalogues having radio flux measurements at the same $1.4 \mathrm{GHz}$ frequency. This way we benefit from repeated observations from both catalogues, as they give more accurate positions and fluxes and more important, reveal large differences between the two measured fluxes, thus allowing to establish radio variability. As a result, 79,382 radio variables have been revealed, including 6301 with flux differences at $1.4 \mathrm{GHz}$ larger than 15 mJy, 1917 with flux differences $>45$ mJy and 260 with flux differences $>200$ mJy. By using a special technique, 2425 optically variable objects out of 6301 radio sources have been revealed. 2425 radio sources with both high radio and optical variability into four categories have been divided. 1206 (19\%) out of 6301 radio sources have activity types from available catalogues and $619(25.5 \%)$ out of 2425 radio sources with at the same time radio and optical variability have activity types from available catalogues. In addition, 279 radio sources out of 2425 have high variability in optical range. We have established their activity types when available. The IR fluxes and colours for the 6301 variable radio sources have been studied. Colour-colour diagrams show that most of the "unknown" sources are galaxies. The activity types for $110(42 \%)$ out of 260 extremely high variable radio sources also have been retrieved (Abrahamyan et al. (2018)). 
The analysis of blazars' parameters from BZCAT leads to a conclusion that they do not have the same properties. The preliminary criterion to include an object in the catalog was the strong radio emission; however, two type of radio sources were selected: BL Lacertae (BLL) objects and Flat Spectrum Radio Quasars (FSRQ). As a number of properties are typical of blazars (strong radio emission, optical variability, continuum optical spectra without lines, polarization, high luminosity, etc.), using the optical data, we investigate them to clarify which property plays the most significant role in their classification as blazars. We found that $60 \%$ of blazars have optical variability. We use a technique developed based on POSS1 and POSS2 photometry and group the variability into extreme, strong, medium, and low classes. In the optical range, 51 blazars have powerful variability (extreme variables), and 126 are high variables. In addition, $63 \%$ of blazars have detected radiation in X-ray and $28 \%$ have detected radiation in gamma rays. We give the average statistical characteristics of blazars based on our analysis and calculations Abrahamyan et al. (2019).

In the next work we investigate radio properties of active galaxies taken from Véron-Cetty \& Véron (2010) catalogue. The galaxies are limited to magnitudes in the range of $12^{m}-19^{m}$. We have cross-correlated the list with radio catalogues and selected those galaxies, which have data on 6 or more radio fluxes at different wavelengths. As a result, we have 198 galaxies, which satisfying these conditions. Using SDSS DR15, we have obtained 96 spectroscopic identifications out of the 198 objects. After the classification, $85 \%$ of 96 objects have changed their types. Available data on the classification of these objects and our classification showed that $56.7 \%$ of them are Seyfert galaxies. For all the objects we have built radio spectra and estimated radio spectral indices. As a result, we obtain $\bar{\alpha}=-0.6089 \pm 0.056\left(\bar{\alpha}_{\text {Seyfert }}=-0.6013 \pm 0.027, \bar{\alpha}_{L I N E R}=-0.5955 \pm 0.025, \bar{\alpha}_{H I I}=-\right.$ $\left.0.6672 \pm 0.039, \bar{\alpha}_{\text {Comp. }}=-0.7128 \pm 0.043\right)$. We discuss the radio properties of active galaxies based on their radio spectral indices. Using the spectra from the SDSS catalog, 96 objects were studied and detailed types of activity were given for them. For more confident classification we used three diagnostic diagrams and direct study of the spectra. As a result, we have changed classification for $85 \%$ of these objects (Abrahamyan (2020) and Abrahamyan et al. (2020)).

\section{Acknowledgements}

I would like to thank my esteemed supervisor - Dr. Areg Mickaelian for his invaluable supervision, support and tutelage during the course of my $\mathrm{PhD}$ degree.

This work was partially supported by the RA MES State Committee of Science, in the frames of the research project No. 15T-1C257. This work was made possible in part by a research grant from the Armenian National Science and Education Fund (ANSEF) based in New York, USA (grants astroex-4193 and astroex-4195).

\section{References}

Abrahamyan H. V., 2020, Astronomische Nachrichten, 341, 6-7, 703

Abrahamyan H. V., Mickaelian A. M., Knyazyan A. V., 2015, Astronomy and Computing, 10, 99

Abrahamyan H. V., Mickaelian A. M., Paronyan G. M., Mikayelyan G. A., Gyulzadyan M. V., 2018, Astronomy and Computing, 25,176

Abrahamyan H. V., Mickaelian A. M., Paronyan G. M., Mikayelyan 2019, Astronomische Nachrichten, $340,5,437$

Abrahamyan H. V., Mickaelian A. M., Paronyan G. M., Mikayelyan 2020, Astrophysics, 63, 3, 322

Hovatta T., et al., 2014, Mon. Not. R. Astron. Soc. , 439, 690

Véron-Cetty M. P., Véron P., 2010, Astron. Astrophys. , 518, A10 